\title{
6 INOVAÇÕES NA FORMAÇÃO EM SAÚDE: O PROGRAMA DE EDUCAÇÃO PELO TRABALHO - SAÚDE MENTAL ${ }^{1}$
}

| Malena Rosa² Ândrea de Souza ${ }^{3}$; Ana Lúcia Abrahãón ; Dalvani Marques ${ }^{5}$ |

\section{RESUMO}

CONTEXTO: A qualificação dos profissionais de saúde ainda se constitui em um grande desafio para o fortalecimento da atenção psicossocial e do Sistema Único de Saúde (SUS). É preciso formar profissionais com habilidades e competências para a implementação de um cuidado em rede, cujo foco seja o usuário.

OBJETIVOS: Avaliar se o Programa de Educação pelo Trabalho (PET-Saúde) na saúde mental tem se constituído em uma estratégia de educação permanente para os profissionais.

METODOLOGIA: Estudo descritivo com abordagem qualitativa, realizado nos serviços de saúde mental que sediam a experiência do PET no município de Niterói, Rio de Janeiro. Os participantes da pesquisa foram preceptores do PET-Saúde Mental. Para a coleta de dados foram realizadas entrevistas semiestruturadas. O tratamento das informações foi ancorado na análise temática. Esta pesquisa foi aprovada pelo CEP sob n ${ }^{\circ} 209.321$.

RESULTADOS: Foram entrevistados nove preceptores. A partir da análise do material foram identificadas três categorias analíticas: O PETSaúde Mental como dispositivo de educação permanente; Ações de educação permanente presentes no PET-Saúde Mental na perspectiva dos preceptores; Potencialidades do PET-Saúde Mental como dispositivo de educação permanente.

CONCLUSÕES: O PET-Saúde Mental tem se constituído em um disposto potente de novas modalidades de educação permanente em saúde. Este impele para um novo posicionamento dos profissionais diante de seus processos de formação em e nos serviços, convocando-os a ocuparem a centralidade nos processos de educação no cotidiano dos serviços.

PALAVRAS-CHAVE: Saúde mental; Educação continuada; Saúde mental; Ensino em saúde

\section{RESUMEN}

\section{“Innovaciones de la formación en salud: Programa de educación por el trabajo - salud mental"}

CONTEXTO: La calificación de los profesionales de salud es todavía un gran reto para el fortalecimiento del cuidado psicosocial y del Sistema Único de Salud (SUS). Es necesario formar profesionales con habilidades y competencias para implementar un cuidado en red enfocado en el usuario.

OBJETIVO: Evaluar si el Programa de Educación por el Trabajo (PETSalud) en la salud mental es presentemente una estrategia de educación permanente para los profesionales.

METODOLOGÍA: Estudio descriptivo con abordaje cualitativo, realizado en los servicios de salud mental sedes de la experiencia del PET en el municipio de Niterói, Rio de Janeiro. Los preceptores del PET-Salud Mental fueron los participantes de la investigación. Para recolectar los datos se realizaron entrevistas semiestructuradas. El tratamiento de las informaciones se basó en el análisis temático. Esta investigación fue aprobada por el CEP con el nº 209.321.

RESULTADOS: Se entrevistaron a nueve preceptores. A partir del análisis del material fueron identificadas tres categorías analíticas: El PET-Salud Mental como dispositivo de educación permanente; Acciones de educación permanente presentes en el PET-Salud Mental en la perspectiva de los preceptores; Potencialidades del PET-Salud Mental como dispositivo de educación permanente.

CONCLUSIÓN: El PET-Salud Mental se ha convertido en un dispositivo potente de nuevas modalidades de educación permanente en salud. Este impele hacia un nuevo posicionamiento de los profesionales frente a sus procesos de formación en los servicios, convocándolos para que ocupen la centralidad en los procesos de educación en el cotidiano de estos.

\section{DESCRIPTORES: Educación en salud; Salud mental; Educación} continua

\begin{abstract}
"Innovation in health education: Education programme through work - mental health"

BACKGROUND: The qualification of health professionals still remains a major challenge for the reinforcement of psychosocial care and the Sistema Único de Saúde (SUS) (Unified Health System). It is necessary to train students in order to provide them the skills and competences for the
\end{abstract} implementation of a user-focused care network.

AIM: To confirm if the Program of Education through Work (PETHealth) on mental health has resulted in a permanent education strategy for practitioners.

METHODS: This is a descriptive study performed by means of a qualitative approach, carried out in mental health service units that host the PET experience in Niterói, Rio de Janeiro. The participants were trainers of PET-Mental Health. Data collection was performed by means of semistructured interviews. Information processing was based in the thematic analysis. This study was approved by the CEP under No. 209,321.

RESULTS: Nine preceptors were interviewed. From the analysis of the material, three analytical categories were identified: PET-Mental Health as a continuing education device; permanent education actions present in the PET-Mental Health from the perspective of preceptors; potentialities of PET-Mental Health as a continuing education device.

CONCLUSION: PET-Mental Health has proven to be a powerful tool for the provision of improved types of permanent health education. It boosts the positioning of professionals in the training processes and service provision, summoning them to occupy a key role in education processes that happen in daily services.

\section{KEYWORDS: Mental health; Continuing education; Health} education

Submetido em 22-02-2016 Aceite em 30-05-2016

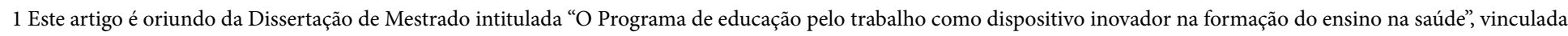
ao Programa de Mestrado de Ensino na Saúde da Universidade Federal Fluminense.

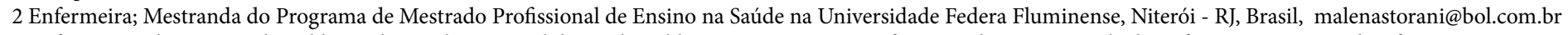

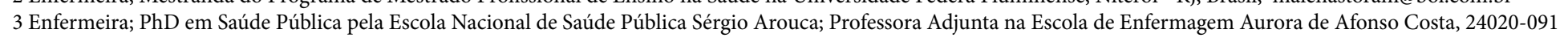
Niterói - RJ, Brasil, andriacz@ig.com.br

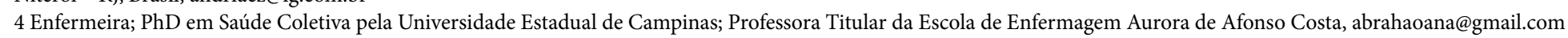
5 Enfermeira; Professora Adjunta na Escola de Enfermagem da Universidade Estadual de Campinas, 13083-970 Campinas - SP, Brasil, marquesdal@yahoo.com.br

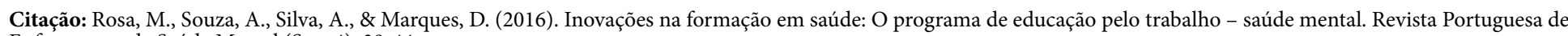
Enfermagem de Saúde Mental (Spe. 4), 39-44. 


\section{INTRODUÇÃO}

A formação profissional em saúde mental é um processo em constante construção, sendo um tema frequentemente debatido com o intuito de criar estratégias e desenvolver ações capazes de qualificar profissionais para uma prática diferenciada, que tenha o sujeito como seu principal foco de ação e que seja mais condizente com os princípios do Sistema Único de Saúde (SUS).

Ao mesmo tempo, o modelo de atenção em saúde mental preconizado pelo SUS vem se consolidando através de muitas mudanças de paradigmas e buscando cada vez mais atender a população de maneira integral, o que exige profissionais de saúde envolvidos com o contexto histórico-social dos usuários e que possam atuar como transformadores do processo saúde-doença. Para tanto, o Ministério da Saúde implementou uma nova estratégia para a formação de profissionais, um programa que tem como um dos seus objetivos a reorientação da formação profissional, objetivando profissionais mais qualificados para a atuação no SUS: o Programa de Educação pelo Trabalho na Saúde (PET-Saúde).

O PET-Saúde prevê a atuação em redes temáticas de atenção: atenção psicossocial, doenças crônicas não transmissíveis, rede cegonha, entre outras. Cada grupo Pet-Saúde Mental é composto por um coletivo formado por professores universitários, alunos de graduação e profissionais dos serviços. O programa prevê a participação de todas as categorias profissionais concernidas ao campo da saúde e tem como uma das suas principais características a interdisciplinaridade.

De acordo com Morais, Jales, Silva e Fernandes (2012) a dinâmica interdisciplinar do programa tende a favorecer a apreensão de um novo conhecimento para a produção de outras práticas que intencionam a articulação do saber com as necessidades dos sujeitos.

A experiência interdisciplinar além de enriquecer o saber com novas formas de cooperação e comunicação entre os profissionais e entre estes e o usuário, dá ao profissional de saúde condições de perceber o sujeito como um todo, necessitando, assim, de uma visão mais ampla, que ultrapasse a sua especificidade profissional, e que caminhe na direção da compreensão das implicações sociais decorrentes de sua prática (Loch-Neckel, Seemann, Eidt, Rabiske e Crepaldi, 2000).

Uma outra premissa do PET é tomar o mundo do trabalho como espaço de aprendizado. Nesse sentido, o programa encontra-se contextualizado na Política de Educação Permanente em Saúde que defende o conceito de aprendizagem-trabalho como sendo aquela que acontece no cotidiano das pessoas e dos serviços.
Propõe que o processo de educação dos trabalhadores da saúde se faça a partir da problematização do processo de trabalho, pautando a necessidade de formação e desenvolvimento dos trabalhadores pelas necessidades de saúde das pessoas.

Desse modo, um bom ponto de partida para o desenvolvimento de uma estratégia consistente de educação permanente seria conhecer a realidade dos serviços e, a partir das experiências já adquiridas pelos profissionais, levantar sugestões e promover espaços de troca e discussões relativas ao processo de trabalho. É preciso valorizar o conhecimento produzido no próprio cotidiano do serviço, mas acima de tudo estimular a reflexão acerca do mesmo, de forma a superar as possíveis dificuldades encontradas e possibilitar novas ações que promovam uma atenção cada vez mais integral aos usuários dos serviços de saúde mental.

A experiência é destacada por Abrahão e Merhy (2014) como um elemento dinamizador da formação, que implica colocar-se à disposição do exercício de apreender com e no mundo do trabalho, enquanto um campo essencialmente micropolítico. Enfatizam que a formação nos convoca a experimentar durante o cuidar, durante $o$ ato do trabalho, sensações e afetos produzidos no cuidado.

A educação permanente cria espaços de reflexão para que os profissionais possam repensar sua prática, entender os processos de trabalho no qual estão inseridos e rever condutas, de modo a buscar novas estratégias de intervenção, superação de dificuldades individuais e coletivas no trabalho.

Por ser uma estratégia inovadora e recente no campo da saúde mental e que, segundo Rodrigues, Souza e Pereira (2012) está sendo construída por sujeitos sociais que optaram por uma proposta dinâmica de formação por meio do trabalho em saúde e, utilizando a educação permanente como ferramenta, busca consolidar uma formação mais condizente com a política nacional de saúde mental, rompendo, inclusive, com padrões históricos e tradicionais do processo de ensino-aprendizagem.

O objetivo deste estudo consiste em avaliar se o PetSaúde tem se constituído em uma possibilidade de educação permanente dos profissionais/preceptores dos serviços de saúde mental do município de Niterói, RJ, Brasil. 


\section{METODOLOGIA}

Este estudo consiste em uma pesquisa descritiva com abordagem qualitativa. O cenário deste estudo foram os serviços da rede pública de saúde mental do município de Niterói que são vinculados ao PET-Saúde Mental.

Os participantes da pesquisa foram os profissionais de saúde que atuavam como preceptores do Pet-Saúde Mental. Os critérios de inclusão adotados foram: profissionais de saúde que atuavam nos serviços da rede pública de saúde mental de Niterói como preceptores do PET. Foram excluídos os profissionais que atuavam como voluntários do PET, os servidores que se encontravam em licença e ou férias durante a realização da pesquisa.

Para coleta de dados foram realizadas entrevistas semiestruturadas com oito preceptores. Estas foram realizadas nos serviços de saúde mental que esses profissionais atuavam. Para o tratamento dos dados optou-se por realizar a análise temática.

Esta pesquisa foi submetida ao Comitê de Ética em Pesquisa do Hospital Universitário Antônio Pedro da Universidade Federal Fluminense e recebeu parecer favorável nº 862.452.

\section{RESULTADOS}

Foram entrevistados oito profissionais que atuavam nos serviços de saúde mental do município de Niterói e que participavam como preceptores do PET-Saúde Mental. Dos participantes do estudo, 50\% pertenciam ao sexo feminino e $50 \%$ ao masculino. Todos trabalhavam na rede pública de atenção psicossocial do município, sendo que dois atuavam no Centro de Atenção Psicossocial (CAPS) álcool e drogas, um atuava no CAPS para infância e adolescentes, um atuava na gestão, dois trabalhavam no setor de álcool e drogas, um no setor de emergência do hospital psiquiátrico e um atuava no consultório na rua.

Os dados foram coletados nos locais de trabalho dos preceptores por meio de entrevista semiestruturada, nos meses de junho a agosto de 2015.

A partir da análise dos dados foram identificadas três categorias analíticas: O PET-Saúde Mental como dispositivo de educação permanente; Ações de educação permanente presentes no PET-Saúde Mental na perspectiva dos preceptores; Potencialidades do PETSaúde Mental como dispositivo de educação permanente.
O PET como Dispositivo de Educação Permanente

Uma das prerrogativas do PET-Redes de Atenção Psicossocial é incentivar a formação e qualificação técnica de profissionais de saúde mais adequadas às necessidades do SUS, fomentando seu espírito crítico para a construção de um cuidado cada vez mais integrado e pautado na indissociabilidade entre ensino-serviçocomunidade.

Entendendo os serviços de saúde como campos potentes para a formação, seja pelos desdobramentos produzidos pelo encontro dos alunos com os usuários e cenários das unidades de saúde, mas principalmente na troca estabelecida na relação aluno-preceptor e demais profissionais dos serviços é que o PET-Saúde pode ser apontado como um dispositivo de educação permanente.

Do universo de oito preceptores do programa entrevistados, $100 \%$ consideraram o PET uma proposta de educação permanente.

"Os profissionais são incentivados a trazerem suas questões para o debate com seus colegas possibilitando troca de saberes, estranhamento e desacomodação de saberes". (P4)

"Sim. Pois é um contínuo de busca por respostas e problematizações das práticas cotidianas de cuidado na rede". (P9)

- Ações de educação permanente presentes no PETSaúde Mental na perspectiva dos preceptores

$\mathrm{Na}$ saúde mental, grande parte do processo de trabalho se dá nos espaços de reunião de equipe e supervisão institucional e isso aparece também no discurso dos preceptores participantes desta pesquisa.

Dentre as ações de educação permanente apontadas pelos preceptores, destacam-se: as reuniões mensais do PET com tutores, preceptores e alunos; preceptoria em serviço; os espaços de reunião de equipe e supervisão institucional; a busca de informações e pesquisa de material bibliográfico.

"Encontro permanente entre os profissionais na busca de articular a prática e lugar de discussão teórica e clínica, tendo o ensino e a formação o seu objetivo”. (P4) "Supervisões clínico institucionais... encontros de preceptoria e encontros mensais com todos os alunos, preceptores e tutores". (P4)

- Potencialidades do PET como dispositivo de educação permanente

Mais do que descrever as ações que podem ser consideradas como de educação permanente, este estudo buscou avaliar se o PET é um dispositivo em potencial para que o trabalho de educação permanente aconteça. 
Existem processos de formação que surgem no ato da preceptoria, em cada encontro com a prática, com as dificuldades, com os desafios; processos que não estão previamente determinados. E é nesse cenário do imprevisto, do novo, do que surge da vivência de cada profissional com o exercício da preceptoria que se pretende qualificar as potencialidades do PET para a educação permanente.

"A forma como se propõe a transmissão do conhecimento e a valorização da experiência em ato é um dos principais pontos que caracteriza o PET como uma educação permanente". (P2)

\section{DISCUSSÃO}

Através da parceria estabelecida entre Ministério da Saúde e Ministério da Educação com o objetivo de estimular mudanças curriculares nos cursos de graduação da área de saúde, de modo a formar profissionais com perfil mais adequado às necessidades do SUS, surgiram nos últimos anos alguns programas de destaque do qual o PET-Saúde faz parte.

A partir dos resultados do estudo é possível considerar o PET-Saúde Mental como um dispositivo inovador e potente para a qualificação e educação permanente na saúde.

A educação permanente é um dispositivo importante para a implementação de mudanças no SUS, e também para reforçar a relação das ações de formação com a gestão do sistema dos serviços, com o trabalho da atenção à saúde. O PET como dispositivo da educação permanente é um provocador de mudanças nas práticas de saúde, visto que proporciona reflexão crítica sobre o cotidiano do trabalho de profissionais na rede de serviços. O PET-Saúde: Redes de Atenção Psicossocial lançou mão de estratégias focadas no cenário da prática, no processo de ensino-aprendizagem que se dá na rede de serviços, envolvendo o docente, o estudante, a equipe de saúde do serviço e os usuários do SUS para incitar processos de educação permanente nos serviços.

Entendendo que para prestar um cuidado global ao usuário é preciso conhecer os fatores determinantes do processo saúde-doença, faz-se necessária uma atuação em saúde pautada na integralidade e cada vez mais em estratégias interdisciplinares que possam atender às diferentes demandas envolvidas na promoção da saúde de um indivíduo (Muniz, Tavares, Abrahão e Sousa, 2015).
Sendo assim, o PET-Saúde abrange diversos profissionais e busca a integração entre eles, por meio de atividades desenvolvidas na prática e que possam promover uma vivência interdisciplinar para os profissionais de distintas formações.

A experiência do PET-Saúde Mental na promoção de melhor articulação entre os serviços de saúde, a universidade e a comunidade, tem sido relatada em diversos estudos, assim como as dificuldades e desafios para sua implantação. Destaca-se entre eles a desconstrução de uma visão fragmentada do processo de cuidado por parte dos profissionais.

A fragmentação do saber e a pouca articulação entre os profissionais são apontadas como dificuldades relevantes a serem superadas para que se possa construir processos de trabalho interdisciplinares.

Abrahão e Merhy (2014) destacam a necessidade de aumentar o número de intervenções realizadas conjuntamente por profissionais de duas ou mais áreas e identifica a rotina de serviços e a falta de cultura de alguns trabalhadores para desenvolver atividades multiprofissionais, engendradas por uma concepção interdisciplinar, como os principais fatores a serem reformulados para que se tenha processos de cuidado mais condizentes com as demandas dos usuários. Sugere ainda que a criação de mais oportunidades de encontro entre as áreas envolvidas possibilitaria uma vivência interdisciplinar entre eles.

A educação permanente é um dispositivo importante nesse cenário, já que se trata de um dispositivo para mudanças nas práticas de saúde, baseadas na reflexão crítica sobre o cotidiano do trabalho de profissionais na rede de serviços, considerando a integralidade como princípio da formação profissional.

O PET-Saúde: Redes de Atenção Psicossocial reúne características para se concretizar como um dos agentes da mudança na maneira de pensar a formação profissional. Tanaka et al. (2012) afirmam que o PET-Saúde é um instrumento para qualificação do serviço e incentiva a integração ensino-serviço-comunidade, a institucionalização das atividades pedagógicas dos profissionais do serviço e o estímulo para a inserção das necessidades do serviço como fonte de produção de conhecimento e pesquisa na universidade.

Com a entrada em cena de docentes e estudantes atuando junto com os trabalhadores, há certamente uma oxigenação, uma motivação que aponta para a alteração na cultura estabelecida no cuidado em saúde. 


\section{CONCLUSÃO}

Na perspectiva de repensar a formação para a atenção psicossocial é que está inserida a proposta do PETSaúde Mental, tendo também como princípio orientador a integralidade. O cuidado integral em saúde requer um olhar interdisciplinar e no PET-Saúde Mental isso se dá não apenas pela observação do trabalho em equipe nos campos de prática, mas também nos encontros dos profissionais com os usuários, onde podem observar a necessidade de se promover uma atenção compartilhada tanto com os demais profissionais quanto com outros dispositivos de uma rede de saúde envolvida no cuidado do usuário.

Para realizar uma prática que atenda à integralidade, o trabalho em equipe precisa ser exercitado desde o processo de formação do profissional de saúde, a partir de estratégias de aprendizagem que favoreçam o diálogo, a troca, a transdisciplinaridade entre os distintos saberes que contribuam para as ações de promoção de saúde no âmbito individual e coletivo. Sendo assim, trabalhar a integralidade desde a formação se faz indispensável para que se tenha uma atenção em saúde centrada no usuário e voltada não apenas para atender as suas demandas, mas também para fazê-lo parte integrante de seu processo de cuidado.

O PET-Saúde Mental tem servido como uma ferramenta para a aproximação das políticas públicas de saúde e a prática dos profissionais em suas próprias áreas de atuação. Contudo, não se pode esquecer dos desafios que impõem essa transformação. Para que de fato sejam criados espaços de aprendizagem através do próprio ambiente de trabalho, é imprescindível a desconstrução de um modelo de formação profissional pautado no tecnicismo, centrado em procedimentos e na doença. Cabe destacar o caráter de continuidade exigido por esse processo, onde cada profissional torna-se protagonista na construção de seu próprio conhecimento e na criação de novos espaços que possibilitem a formação conjunta com outros profissionais.

Trata-se de se permitir aprender de novo, de questionar um fazer que já é dado como certo e repensar se ele pode ser realizado de outra forma. Não é, contudo, tarefa fácil para um profissional, tomar o saber acumulado em sua formação e reinventá-lo ou readaptá-lo ao cotidiano sem que haja um exercício, um incentivo a essa prática, o que pode, muitas vezes, gerar resistência por aqueles que executam o trabalho.
As barreiras impostas à implantação da educação permanente ocorrem também por parte das unidades de saúde mental, devido a pouca organização dos serviços e das escalas dos profissionais de modo a não favorecer a criação de espaços de discussão que possibilitem uma reflexão sobre a prática e a promoção de melhorias no cuidado prestado aos usuários. Fazer caber no cotidiano dos serviços espaços de educação permanente torna-se um desafio face às inúmeras demandas, rotinas e processos de trabalho que dificultam o encontro dos profissionais.

A educação permanente é um processo que impõe uma série de desafios que precisam ser transpostos para garantir sua efetividade. É preciso inserir seus fundamentos na rotina de trabalho e considerar, portanto, as atividades dentro da carga horária contratual do trabalhador.

Nas unidades de saúde mental que integraram o PETSaúde Mental, em função de várias ações de educação permanente colocadas em prática, desde oficinas até os benefícios de ordem material e patrimonial, houve uma desacomodação na cultura estabelecida, ou seja, houve uma provocação positiva no ânimo e no comportamento dos trabalhadores.

O PET Atenção Psicossocial tem proporcionado aos profissionais de saúde mental do município de Niterói uma reflexão prática sobre conceitos que sustentam o campo. Tem-se constituído um espaço de educação permanente no interior dos serviços. Os profissionais atribuem, principalmente, às reuniões de supervisão em serviço para discussão de casos clínicos e às reuniões com os tutores, os espaços privilegiados de educação permanente no cotidiano dos serviços.

\section{IMPLICAÇÕES PARA A PRÁTICA CLÍNICA}

Destacando a importância da formação de profissionais cada vez mais implicados para a adoção de um trabalho articulado em rede e na rede e de acordo com as reais necessidades do SUS, o PET-Saúde: Rede de Atenção Psicossocial, constitui-se em um dispositivo para viabilizar programas de aperfeiçoamento e especialização em serviço dos profissionais de saúde, estimulando sua constante qualificação e tornando-os coprodutores de conhecimentos e práticas que atendam às demandas dos usuários.

O Pet Saúde Mental tem estimulado a reflexão sobre o trabalho que já é desempenhado pelos profissionais e, aproveitando suas experiências, criar novas formas de transmitir informação, reinventar o cenário da prática e articular o cotidiano do serviço. 


\section{REFERÊNCIAS BIBLIOGRÁFICAS}

Abrahão, A., e Merhy, E. (2014). Formação em saúde e micropolítica: Sobre conceitos-ferramentas na prática de ensinar. Interface-Comunicação, Saúde, Educação, 18(49), 313-324. doi: dx.doi.org/10.1590/180757622013.0166

Loch-Neckel, G., Seemann, G., Eidt, H., Rabuske, M., e Crepaldi, M. A. (2009). Desafios para a ação interdisciplinar na atenção básica: Implicações relativas à composição das equipes de saúde da família. Ciência \& Saúde Coletiva, 14(Suppl. 1), 1463-1472. doi: dx.doi. org/10.1590/S1413-81232009000800019

Machado, M., Monteiro, E., Queiroz, D., Vieira, N., e Barroso, M. (2012). Integralidade, formação de saúde, educação em saúde e as propostas do SUS - uma revisão conceitual. Revista Brasileira de Educação Médica, 36(1), 136-140. Disponível em http://www.scielosp. org/pdf/csc/v12n2/a09v12n2.pdf
Morais, R., Jales, L., Silva, M., e Fernandes, S. (2012). A importância do PET-Saúde para a formação acadêmica do enfermeiro. Trabalho, Educação e Saúde, 10(3), 541-551. doi: dx.doi.org/10.1590/S198177462012000300011

Muniz, M., Tavares, C., Abrahão, A., e Souza, A. (2015). A assistência de enfermagem em tempos de reforma psiquiátrica. Revista Portuguesa de Enfermagem de Saúde Mental (13), 61-65. Disponível em http://www. scielo.mec.pt/pdf/rpesm/n13/n13a08.pdf

Rodrigues, A., Souza, S., e Pereira, H. (2012). Rede pet em Feira de Santana: O desafio da integralidade. Revista Baiana de Saúde Pública, 36(3), 651-667. Disponível em http://inseer.ibict.br/rbsp/index.php/rbsp/article/ view/545/0

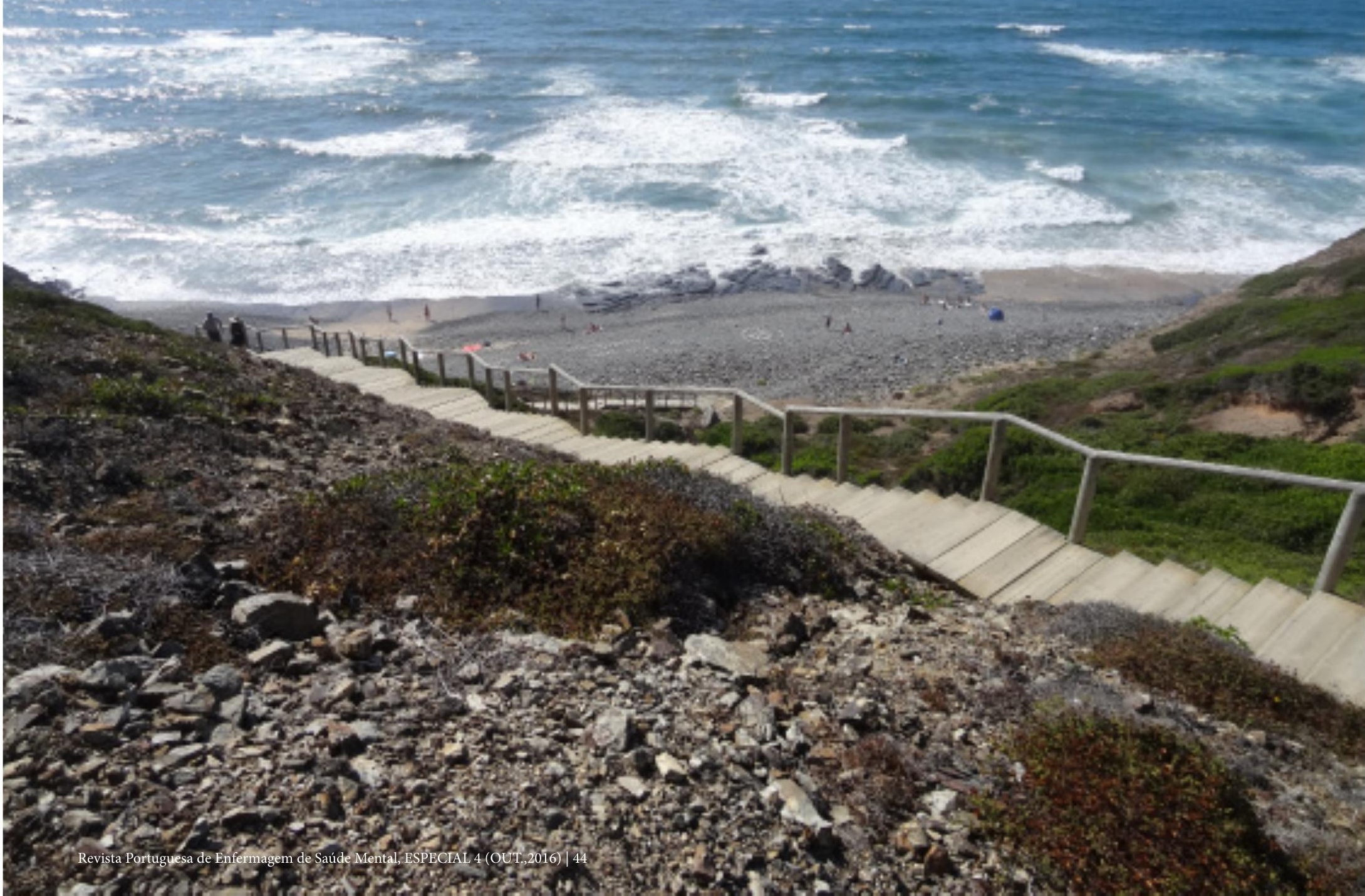

